\title{
An Ultrafast Direct Electron Camera for 4D STEM
}

Debaditya Chatterjee ${ }^{1}$, Jingrui $\mathrm{Wei}^{2}$, Alex kvit ${ }^{3}$, Benjamin Bammes ${ }^{4}$, Barnaby Levin ${ }^{5}$, Robert Bilhorn ${ }^{6}$ and Paul Voyles ${ }^{7}$

${ }^{1}$ University of Wisconsin-Madison, Madison, Wisconsin, United States, ${ }^{2}$ Department of Materials Science and Engineering, University of Wisconsin Madison, Madison, Wisconsin, United States, ${ }^{3}$ University of Wisconsin-Madison, United States, ${ }^{4}$ Direct Electron LP, Pearland, Texas, United States, ${ }^{5}$ Direct Electron, Inc., United States, ${ }^{6}$ Direct Electron LP, San Diego, California, United States, ${ }^{7}$ University of Wisconsin Madison, United States

Four-dimensional scanning transmission electron microscopy (4D STEM) is in the process of revolutionizing imaging and diffraction in electron microscopy. In various modes, 4D STEM offers drastically improved spatial resolution ${ }^{[1]}$, high contrast imaging at low dose ${ }^{[2]}$, and a variety of unique imaging signals ${ }^{[3]}$. The speed and sensitivity of direct detectors is essential to 4D STEM at high spatial resolution, but most of the initial progress was made using adapted detectors originally developed for HRTEM imaging, especially for cryoTEM. Only recently have purpose-built 4D STEM detectors become available ${ }^{[4,5]}$.

We have developed a new, ultrafast direct detector specifically designed for 4D STEM. It is a monolithic active pixel sensor (MAPS) type detector optimized for high frame rate readout and capable of measuring large currents without saturating. The physical sensor is $1024 \times 1024$ pixels, with $15 \mu \mathrm{m}$ pixel size. At full pixel resolution, the detector exceeds 2,100 frames per second (fps) or $\sim 2,200$ megapixels per second. Higher frame rate is achieved by reducing the number of pixels per frame through sub-area readout, up to an anticipated maximum in excess of $100,000 \mathrm{fps}$ at $256 \times 64$ pixels. Sub-area readout can be configured in the acquisition software, enabling the user to trade pixel count for frame rate to suit various applications and imaging modes. The detector can either send or receive a hardware trigger signal to synchronize the high speed frame rate with a scan generator.

Fig. 1 summarizes the basic camera performance. On-chip correlated double sampling (CDS) subtracts the reset noise of each pixel in each frame, increasing the signal to noise ratio (SNR) of the detector. Fig. 1a shows that in rolling shutter readout mode with on-chip CDS enabled, the root-mean-squared pixel value in the absence of incident electrons is just 1.32 analog-digital units (ADU). The signal from $200 \mathrm{kV}$ primary electrons has a mean intensity of 266 ADU and a most probable intensity of 110 ADU, corresponding to a mean single-electron SNR of $\sim 200$ and a most probable single-electron SNR of $\sim 80$. The large pixel size increases performance, especially at low pixel counts and high speed. Fig. 1b shows that the mean detection event size for $200 \mathrm{kV}$ electrons is 2.5 pixels, which yields the modulation transfer function (MTF) in Fig. 1c, exceeding 0.6 at half Nyquist in integration mode. As is the case with other MAPS detectors, electron counting can be applied for images with sufficient sparsity to increase MTF and normalize the signal of each detected electron.

Fig. 2 shows initial 4D STEM data, acquired at 1,800 fps with the full $1024 \times 1024$ pixel readout, from a [100] $\mathrm{SrTiO}_{3}$ sample with a 23.4 mrad convergence angle, $39 \mathrm{pA}$ probe and a maximum collection angle of $34 \mathrm{mrad}$. Fig. 2a is a typical single CBED pattern from the dataset. Fig. 2b is a synthetic BF image showing the $\mathrm{SrTiO}_{3}$ lattice, which shows the $\mathrm{Sr}$ and $\mathrm{Ti}$ sublattices clearly, but does not have the resolution 
or sensitivity at this speed to resolve the O. Fig. 2c is the 90 degree rotation symmetry STEM image ${ }^{[6]}$, showing clear, sharp maxima at the positions of the atomic columns.

In high-gain mode, the new detector saturates at 10 electrons per pixel per frame. At 100,000 fps, this exposure rate corresponds to a saturation current of $160 \mathrm{fA} /$ pixel, which is too low to simultaneously measure high-angle scattering and the central beam of a 4D STEM convergent beam electron diffraction pattern. Therefore, the camera is designed with a low gain mode, which will have reduced SNR for single electrons but a saturation value of $>130$ electrons per pixel per frame, which at 100,000 fps is $\sim 2 \mathrm{pA} / \mathrm{pixel}$, sufficient to measure the central beam without saturation even at very short camera length. The camera also has a "best of both worlds" high dynamic range (HDR) mode, in which the same exposure is read in low- and high-gain mode, resulting in high SNR for weak signals and high saturation for strong signals, at the cost of a $2 \times$ reduction in maximum framerate. Combined with a recently developed HDR counting method which combines counting in the sparse dark field regions with integration in bright regions of each frame $^{[7]}$, HDR mode should be well-suited to the large-angle 4D STEM required by ptychography and center-of-mass imaging ${ }^{[3]}$.

The ultrafast camera will enable 4D STEM at the same speed as current conventional STEM (10 $\mu$ s / probe position). This speed is sufficient to outrun many instrument instabilities, so the technologies developed to improve SNR and reduce spatial distortions in conventional STEM, like non-rigid registration $^{[8]}$ and RevSTEM ${ }^{[9]}$ will become applicable to 4D STEM, pointing toward high precision 4D STEM. Ultrafast acquisition will also enable time-resolved 4D STEM, with the time between entire 4D STEM acquisitions as little as $0.2 \mathrm{~s}$ for small, $128 \times 128$ probe position data sets. This capability will be used for electron correlation microscopy ${ }^{[10]}$ measurements of atomic dynamics in supercooled liquids and for ptychographic movies of defects in materials at elevated temperature.

The camera is installed on a $\mathrm{C}_{\mathrm{S}}$-corrected Titan STEM sited in the University of Wisconsin-Madison Nanoscale Imaging and Analysis Center, a user facility open to all scientists from around the world. Development of the ultrafast camera was supported by the Wisconsin MRSEC (DMR-1720415). Direct Electron acknowledges support from the US Department of Energy, Office of Science, grant DESC0018493.
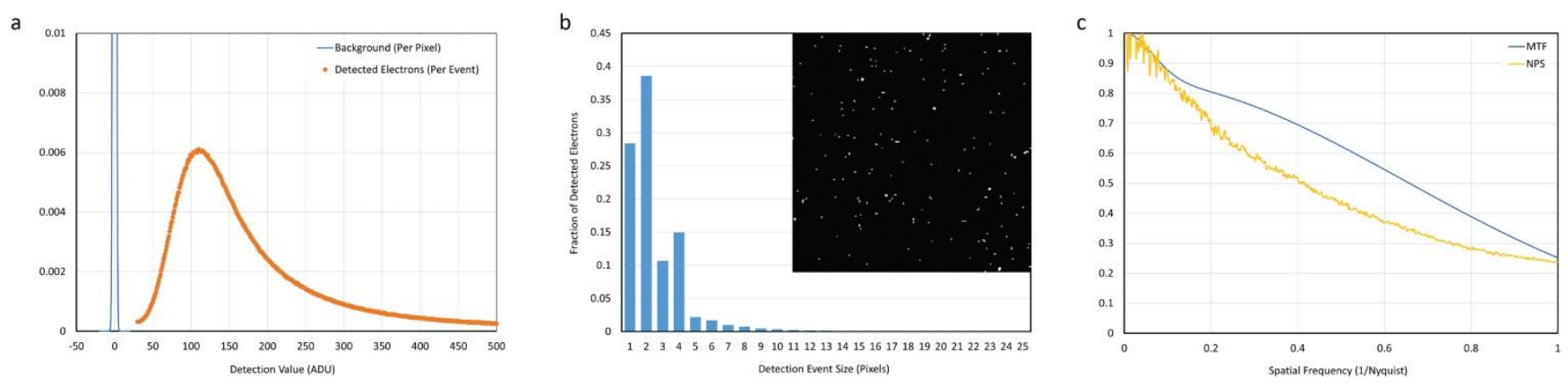

Figure 1. Figure 1: Performance of rolling shutter high-gain mode at $1800 \mathrm{fps}$. (a) Histograms of background noise per pixel per frame in the absence of incident electrons (blue) and the total signal deposited in the detector by incident $200 \mathrm{kV}$ primary electrons (orange). (b) A histogram of the size of each detection event on the sensor. The inset shows an example region of a frame exposed to a low beam intensity so that single electron events are well separated. (c) The modulation transfer function (MTF) and noise power spectrum (NPS) for $200 \mathrm{kV}$ electrons. 

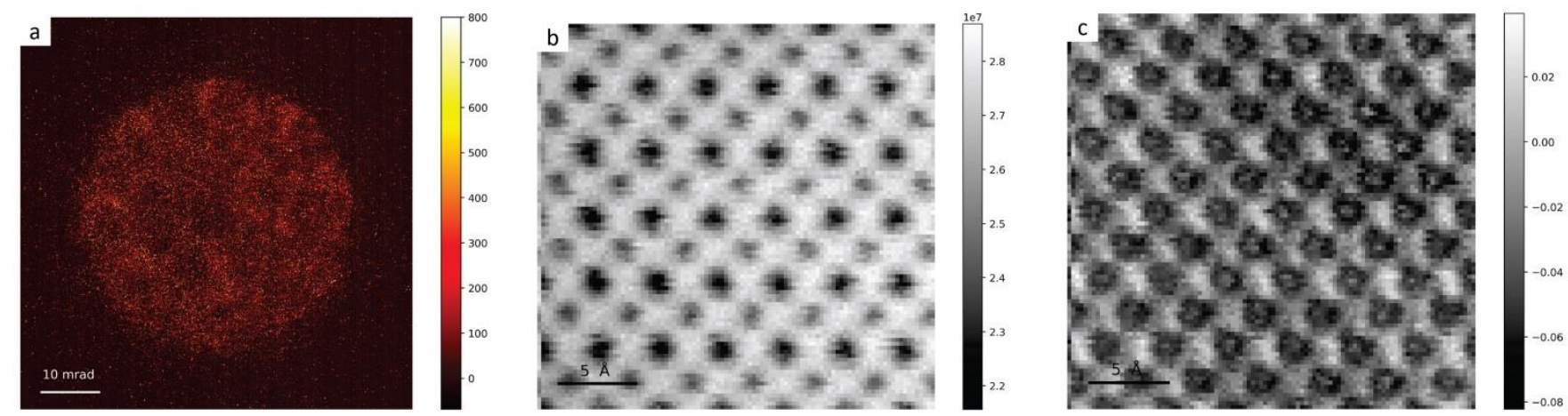

Figure 2. Figure 2: 4D STEM data acquired with the new camera from a SrTiO3 sample at $\sim 1,800 \mathrm{fps}$. (a) A typical single CBED pattern. (b) A synthetic BF STEM image. (c) A 90 degree symmetry STEM image. The color scales in (a) and (b) are in detector counts. The color scale in (c) is the value of the symmetry coefficient.

\section{References}

[1] Y. Jiang, Z. Chen, Y. Han, P. Deb, H. Gao, S. Xie, P. Purohit, M. W. Tate, J. Park, S. M. Gruner, V. Elser, D. A. Muller, Nature 2018, 559, 343.

[2] L. Zhou, J. Song, J. S. Kim, X. Pei, C. Huang, M. Boyce, L. Mendonça, D. Clare, A. Siebert, C. S. Allen, E. Liberti, D. Stuart, X. Pan, P. D. Nellist, P. Zhang, A. I. Kirkland, P. Wang, Nat. Commun. 2020, $11,2773$.

[3] C. Ophus, Microsc. Microanal. 2019, 25, 563.

[4] H. Ryll, M. Simson, R. Hartmann, P. Holl, M. Huth, S. Ihle, Y. Kondo, P. Kotula, A. Liebel, K. Müller-Caspary, A. Rosenauer, R. Sagawa, J. Schmidt, H. Soltau, L. Strüder, J. Instrum. 2016, 11, P04006.

[5] M. W. Tate, P. Purohit, D. Chamberlain, K. X. Nguyen, R. Hovden, C. S. Chang, P. Deb, E. Turgut, J. T. Heron, D. G. Schlom, D. C. Ralph, G. D. Fuchs, K. S. Shanks, H. T. Philipp, D. A. Muller, S. M. Gruner, Microsc. Microanal. 2016, 22, 237.

[6] M. Krajnak, J. Etheridge, Proc. Natl. Acad. Sci. 2020, 117, 27805.

[7] B. D. A. Levin, C. Zhang, B. Bammes, P. M. Voyles, R. B. Bilhorn, Microsc. Anal. 2020, 34(1), 20.

[8] J. K. R. Weber, J. G. Abadie, A. D. Hixson, P. C. Nordine, G. A. Jerman, J. Am. Ceram. Soc. 2004, $83,1868$.

[9] A. B. Yankovich, B. Berkels, W. Dahmen, P. Binev, S. I. Sanchez, S. a. Bradley, A. Li, I. Szlufarska, P. M. Voyles, Nat. Commun. 2014, 5, 4155.

[10] X. Sang, J. M. Lebeau, Ultramicroscopy 2014, 138, 28.

[11] L. He, P. Zhang, M. F. Besser, M. J. Kramer, P. M. Voyles, Microsc. Microanal. 2015, $21,1026$. 